OPEN ACCESS

Edited by:

Jack Tsao,

University of Tennessee Health

Science Center (UTHSC),

United States

Reviewed by:

Veronik Sicard,

Children's Hospital of Eastern Ontario

(CHEO), Canada

Julie Wilson,

Children's Hospital Colorado,

United States

*Correspondence:

Melissa D. Stockbridge

md.stockbridge@jhmi.edu

Specialty section:

This article was submitted to Neurotrauma,

a section of the journal

Frontiers in Neurology

Received: 05 November 2021

Accepted: 10 January 2022

Published: 14 February 2022

Citation:

Stockbridge MD, Keser $Z$ and Newman RS (2022) Concussion in Women's Flat-Track Roller Derby.

Front. Neurol. 13:809939.

doi: 10.3389/fneur.2022.809939

\section{Concussion in Women's Flat-Track Roller Derby}

\author{
Melissa D. Stockbridge ${ }^{1,2 *}$, Zafer Keser ${ }^{2,3}$ and Rochelle S. Newman ${ }^{1}$ \\ ${ }^{1}$ Department of Hearing and Speech Sciences, University of Maryland, College Park, MD, United States, ${ }^{2}$ Department of \\ Neurology, Johns Hopkins University School of Medicine, Baltimore, MD, United States, ${ }^{3}$ Department of Neurology, Mayo \\ Clinic, Rochester, MN, United States
}

Concussions are common among flat-track roller derby players, a unique and under-studied sport, but little has been done to assess how common they are or what players can do to manage injury risk. The purpose of this study is to provide an epidemiological investigation of concussion incidence and experience in a large international sampling of roller derby players. Six hundred sixty-five roller derby players from 25 countries responded to a comprehensive online survey about injury and sport participation. Participants also responded to a battery of psychometric assessment tools targeting risk-factors for poor injury recovery (negative bias, social support, mental toughness) and players' thoughts and feelings in response to injury. Per 1,000 athletes, 790.98 concussions were reported. Current players reported an average of 2.2 concussions, while former players reported 3.1 concussions. However, groups were matched when these figures were corrected for differences in years of play (approximately one concussion every 2 years). Other frequent injuries included fractures in extremities and upper limbs, torn knee ligaments, and sprained ankles. We found no evidence that players' position, full-contact scrimmages, or flooring impacted number of concussions. However, neurological history and uncorrected vision were more influential predictors of an individual's number of concussions during roller derby than years of participation or age, though all four contributed significantly. These findings should assist athletes in making informed decisions about participation in roller derby, though more work is needed to understand the nature of risk.

Keywords: concussion, cognition, language, roller derby, psychometric assessment

\section{INTRODUCTION}

Concussion is defined as "a pathophysiological disturbance in neurologic function characterized by clinical symptoms induced by biomechanical forces" per American Academy of Neurology (1) and up to one-third result in long-term effects (2). Most research on sports-related concussions has focused on a small subset of sports, often utilizing professional athletes (e.g., the National Football League (3); or college-aged athletes, most of whom tend to be men ${ }^{1}$.

\footnotetext{
${ }^{1}$ The Women's Flat Track Derby Association adopts a gender-expansive view of inclusion in the sport and permits any individual to skate with an associated team "if women's flat track roller derby is the version and composition of roller derby with which they most closely identify." Players were recruited using materials and survey questions that specifically asked about women's roller derby participation. Given this inclusion criterion, we have not excluded players who identified as men on the survey from analysis, nor have we excluded any individual on the basis of reported identity. When describing other authors' findings, we have repeated the terms used by the authors themselves, as we cannot be certain which meaning they intended to convey.
} 
Recent studies have examined sex and gender differences in symptomatology and recovery after concussion. Female athletes with comparable injuries report increased post-concussion symptoms both within and beyond 1 week and longer recovery times $(4,5)$. There is some debate as to whether differences are due to biological sex, gender, or other factors (e.g., access to care) (6) that may covary or co-occur (7). The focus of the current descriptive epidemiological study is roller derby, a sport most often played non-professionally by women beyond collegeage who are often remarkably diverse in body type, sexuality and gender identity, and socioeconomic status $(8,9)$. Because of the decentralized culture of roller derby, it is difficult to make clear estimates of the number and demographic characteristics of active players. The Women's Flat Track Derby Association (WFTDA), the largest of seven main international governing bodies for the sport of flat track roller derby, presently boasts 447 member leagues on six continents, but an estimated 758 leagues exist worldwide amidst the pandemic in 2021 (http:// flattrackstats.com/). Each league in WFTDA is required to have at least 15 skaters, but many leagues have multiple teams, each of which have 10-20 skaters. These factors make roller derby dissimilar from the sports played by women that most frequently are studied in the literature on concussions. However, concussion is one of the most common injuries associated with roller derby (10), along with sprains and torn knee ligaments.

To make informed decisions about their participation in sport and assumption of risk, athletes need to know how common injuries are and what choices they can make that may modify their risk of injury. Prior studies have highlighted the potential for a high incidence of concussion in roller derby, despite remarkably limited work directly addressing the sport from a medical perspective. Lane et al. (11) retrospectively examined 1 year of discharged patients with closed head injury or concussion from the University of Arizona Emergency Department and found that $6.5 \%$ of all sports-related concussions $(N=279)$ were the result of "roller derby, roller skating, and skateboarding." This was greater than sports-related concussion from soccer (5\%). It was only exceeded by cycling (30\%), football (12\%), basketball $(12 \%)$, and horseback riding (8\%), sports studied considerably more often within concussion literature.

The two prior investigations into concussion incidence in roller derby had considerable limitations. Cathorall and Peachey (12) found that $4 \%$ of 1,395 players had a concussion in the past year, but the authors did not consider participation across multiple years. Hoskins \& Hooker (13) compiled 74 responses to a tablet-based survey distributed at the 2014 RollerCon roller derby convention in Las Vegas. They found that $54.1 \%$ of athletes had experienced one or more concussions ( 1 concussion per player over 3.6 years of participation, with $10 \%$ reporting three or more), but noted the need for a larger sample size to better estimate concussion in the sport. The first goal of our work was to reexamine these estimates in a international sample of roller derby players in order to describe injury rates among roller derby athletes more thoroughly.

Minimal information has been published on the modification of injury risk in the sport of roller derby. Typically, we examine both the risk of concussion associated with a given sport generally
(14) and the effects of decisions such as rule changes (1518 ) and the introduction of safety equipment $(19,20)$. The WFTDA dictates safety parameters of the play environment and how concussions may be assessed at the sidelines to determine whether an individual may safely continue to participate. They require all skaters, including skating officials, wear wrist guards, elbow and knee pads, mouth guards, and helmets with a hardprotective shell designed for skating (21). Pauelsen (10), in an undergraduate thesis, surveyed 540 players predominantly in Sweden and other countries in Europe and identified multiple risk factors for increased rates of injury in roller derby, including the number of years played and the number of bouts, or matches, per year, contact scrimmages during training, flooring type, and position, with the highest risk associated with the role of the jammer. The WFTDA recommends playing on sport court or concrete (21). Differences in safety also have been noted among body types and levels of fitness (22). Individual differences in health status and history previously have not been examined as potential contributors to increased risk of injury in roller derby. The second goal of our work was to replicate and elaborate on the findings from Pauelsen to include both sport-related factors and personal factors in order to better understand the nature of injury risk in roller derby.

Finally, given the rich literature examining roller derby psychology and culture (23-31), we used this opportunity to take a first look within the context of roller derby at features of personality, temperament, and affect that are known to carry enhanced risk for poor recovery from injury. Dispositional negativity, the propensity for more frequent, intense, and enduring negative affect (32-35), is a risk factor for poor brain injury recovery (36-38). Individuals with high negativity both experience and report a magnified reactivity to negative events or other stressors, further elevating their overall level of disability and perception of disability (39). In contrast, other personal factors have demonstrated a protective effect against stressor reactivity and poor response to trauma. For example, perceived social support is associated with reduced risk associated with dispositional negativity $(40,41)$. Mental toughness also has garnered attention as a protective factor against poor recovery (42), including in roller derby players (43), where authors have noted a complex relationship between toughness, positive injury psychological response, and physical injury exacerbation.

Taken together, the broader purpose of this research was to examine a large international sampling of roller derby players in order to provide a thorough and updated look into concussion incidence and experience by players of roller derby, a highintensity, under-studied sport.

\section{MATERIALS AND METHODS}

\section{Recruitment}

All work was conducted with the formal approval of the University of Maryland Institutional Review Board. Data were collected remotely through Qualtrics experience management software tools distributed through social media across online roller derby communities. Methods of data collection were designed to mitigate in-person barriers to sampling, such 
as transportation and lengthy in-person interviews. Remote behavioral data collection methods, similar to those proposed here, are increasingly common for studies of concussion and other medical conditions and produce comparable results to in-person testing (44-48).

The survey received 1,489 clicks, between February 9, 2018 and November 30, 2019, from 25 countries, including 47 states of the United States. This captured 665 roller derby players who provided the minimum information necessary to be included in analysis (i.e., roller derby participation and concussion endorsement; Table 1).

\section{Data Collection}

Surveys utilized a mixture of closed- (i.e., yes/no, ratings) and open-ended responses. Respondents were asked about their participation in roller derby and other sports with a closed set of responses ("Yes, I currently participate."; "I have participated previously, but don't currently."; "I have never participated."). They also were asked how many years they participated, what role or positions they typically played, how many bouts they participated in per year, and how many practices they participated in per month. They were asked how often scrimmages were full contact and about flooring in the arena where they practiced most frequently. In addition to an openended prompt to list all other sports athletes participated in, we also asked if respondents participated in any other activities that may involve head impacts or other rough physical activity, and if so, for how many years.

In addition to age and gender identity, participants were asked numerous questions about their medical history and recent medical events. Respondents were given a standard definition of concussion and asked to report if they had had one while playing roller derby in the past 30 days or at all and how it was determined that they did. Individuals who only suspected they had a concussion, but it was not verified sideline or by a medical professional, were noted. Since neither the athlete nor an outside medical professional was able to verify a suspected concussion, these were excluded from analysis (though this makes it possible that true concussions would thus be underestimated). Athletes were asked to describe the incident or incidents, part of head that was hit, and whether they were unconscious and for how long (if known). Athletes provided rich medical histories of both past head injuries and other significant injuries and rated their somatic symptoms using a widely available tool (49). Significant hits and concussions were artificially capped at 100, despite some participants endorsing 500 or more hits they considered significant in their lifetime (impacting $N=22$ [3\%] participants whose estimates were $>100)$. The concept of "significance" in this context was left deliberately subjective, though narratives suggested "significance" generally aligned with requiring medical attention resulting in identifiable injury. They also were asked questions about lifetime learning disability, ADD/ADHD, speech language disorder, fluency disorder, dyslexia, epilepsy, their current medications, and their family history of cognitive or language diagnoses. Finally, we asked questions about menstruation, menopause, and pregnancy.
TABLE 1 | Sample description and demographics by roller derby participation.

\begin{tabular}{|c|c|c|c|}
\hline & $\begin{array}{l}\text { Current } \\
(N=535)\end{array}$ & Past $(N=130)$ & Test statistic \\
\hline Age (years) & $32.66 \pm 7.26$ & $35.48 \pm 7.20$ & $\begin{array}{c}\mathrm{t}(663)=3.99 \\
p<0.001\end{array}$ \\
\hline $\begin{array}{l}\text { Gender (see text } \\
\text { footnote 1) }\end{array}$ & & & $\begin{array}{l}\chi^{2}=1.87 \\
p=0.802\end{array}$ \\
\hline Women & 481 & 120 & \\
\hline Men & 25 & 3 & \\
\hline Non-binary/Other & 29 & 7 & \\
\hline Trans $^{\star}$ & 4 & 2 & \\
\hline Education (years) & $16.11 \pm 1.97$ & $15.97 \pm 1.85$ & $\begin{array}{c}t(632)=0.76 \\
p=0.449\end{array}$ \\
\hline Years of roller derby & $4.56 \pm 2.65$ & $5.81 \pm 4.60$ & $\begin{array}{c}t(150)=2.97 \\
p=0.004^{\dagger}\end{array}$ \\
\hline $\begin{array}{l}\text { Practices per } \\
\text { month }\end{array}$ & $10.12 \pm 6.42$ & $10.02 \pm 4.27$ & $\begin{array}{c}t(660)=0.16 \\
p=0.872\end{array}$ \\
\hline Bouts per year & $9.30 \pm 8.59$ & $12.23 \pm 27.40$ & $\begin{array}{c}t(133)=1.19 \\
p=0.235^{\dagger}\end{array}$ \\
\hline \multicolumn{4}{|l|}{$\begin{array}{l}\text { Concussion } \\
\text { endorsement }\end{array}$} \\
\hline Recent & $31(39)$ & 3 & $\begin{array}{c}\chi^{2}=13.33 \\
p=0.002\end{array}$ \\
\hline History & $384(72)$ & 108(8) & $\begin{array}{l}x^{2}=7.64 \\
p=0.023\end{array}$ \\
\hline None & 79 & 14 & \\
\hline $\begin{array}{l}\text { Concussions per } \\
\text { player }\end{array}$ & $2.16 \pm 1.95$ & $3.08 \pm 2.46$ & $\begin{array}{c}t(155)=3.72 \\
p<0.001^{\dagger}\end{array}$ \\
\hline $\begin{array}{l}\text { Concussions per } \\
\text { player per year }\end{array}$ & $0.63 \pm 0.79$ & $0.67 \pm 0.68$ & $\begin{array}{c}t(573)=0.45 \\
p=0.656\end{array}$ \\
\hline $\begin{array}{l}\text { Significant hits per } \\
\text { player }\end{array}$ & $16.90 \pm 27.02$ & $25.24 \pm 34.14$ & $\begin{array}{c}t(112)=1.51 \\
p=0.134^{\dagger}\end{array}$ \\
\hline Somatic symptoms & $20.00 \pm 15.94$ & $25.83 \pm 18.73$ & $\begin{array}{c}\mathrm{t}(162)=3.16 \\
p=0.002^{+}\end{array}$ \\
\hline BRS (/5) & $3.43 \pm 0.77$ & $3.09 \pm 0.84$ & $\begin{array}{c}t(455)=3.66 \\
p<0.001\end{array}$ \\
\hline $\mathrm{BFI}-2 \mathrm{Ne}(/ 100)$ & $50.32 \pm 10.09$ & $52.41 \pm 10.94$ & $\begin{array}{c}\mathrm{t}(440)=1.69 \\
p=0.091\end{array}$ \\
\hline MSPSS (/7) & $5.63 \pm 1.07$ & $5.41 \pm 1.16$ & $\begin{array}{c}t(85)=1.32 \\
p=0.192^{+}\end{array}$ \\
\hline SMTQ (/42) & $38.22 \pm 4.37$ & $38.65 \pm 3.96$ & $\begin{array}{c}t(85)=1.14 \\
p=0.259^{\dagger}\end{array}$ \\
\hline IEQ (/48) & $21.21 \pm 8.74$ & $25.00 \pm 22.63$ & $\begin{array}{l}t(1)=0.24 \\
p=0.852^{+}\end{array}$ \\
\hline IPQ-R (/5) & $2.99 \pm 0.45$ & $3.12 \pm 0.62$ & $\begin{array}{c}\mathrm{t}(101)=1.73 \\
p=0.087^{\dagger}\end{array}$ \\
\hline
\end{tabular}

All statistics are two-tailed.

${ }^{\dagger}$ Degrees of freedom corrected, as equal variances assumption violated. Data are presented as counts or mean \pm standard deviation. Suspected concussions are included in parentheses. Recency was defined as within 30 days. Trans* individuals were included in statistics of the gender identity that they identified. BRS, Brief Resilience Scale; BFI$2 \mathrm{Ne}$, Big Five Inventory 2 negative emotionality $t$-score; MSPSS, Multidimensional Scale of Perceived Social Support; SMTQ, Sports Mental Toughness Questionnaire; IEQ, Injustice Experience Questionnaire; IPQ-R, adaptation of the Revised IIIness Perception Questionnaire referencing concussion. 


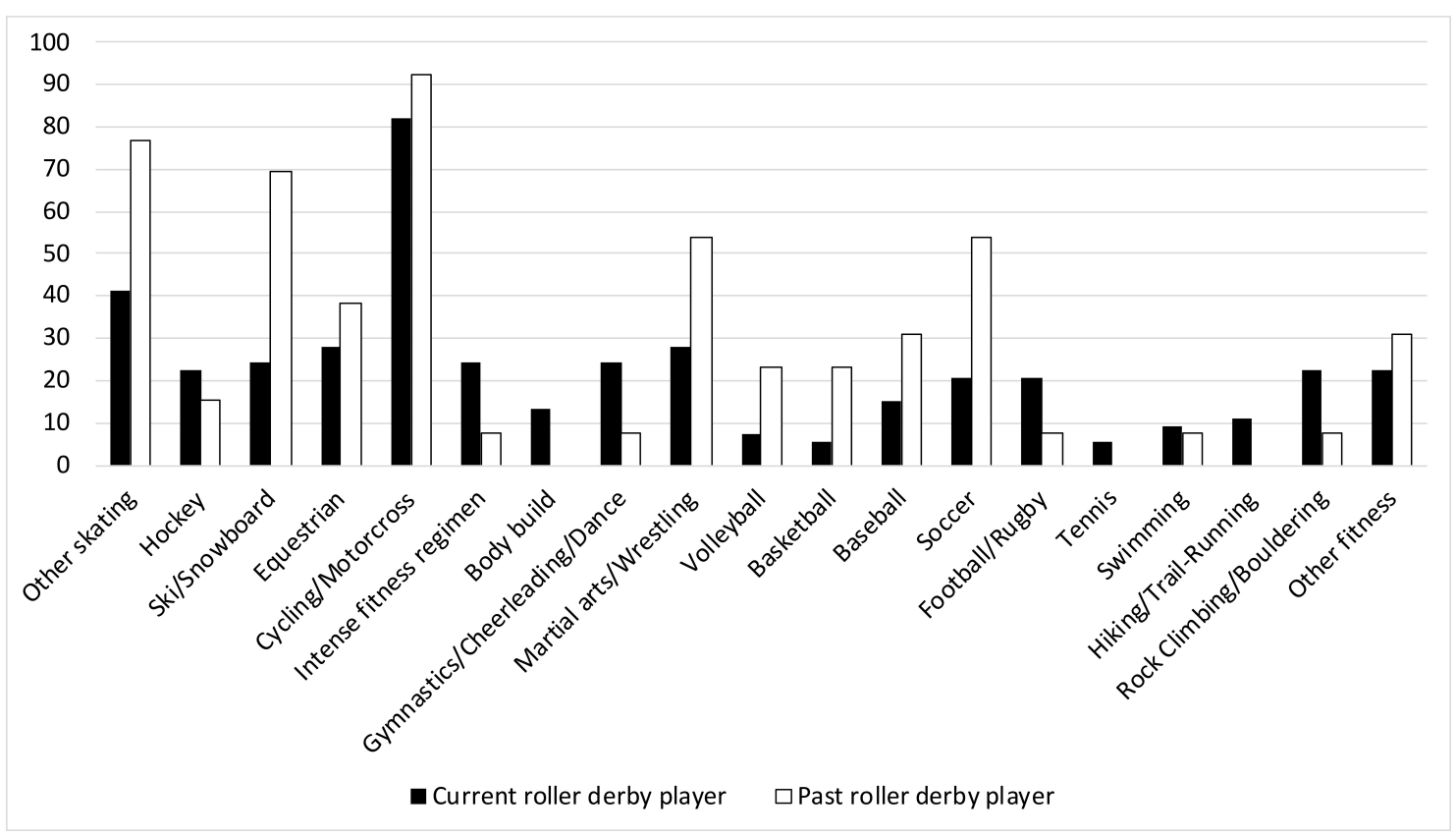

FIGURE 1 | Sport participation outside of roller derby per 1,000 athletes. Other skating: skatepark users, bowl skating, and ice skating. Intense fitness regimens: Tough Mudder, Spartan Races, CrossFit, etc. Other fitness: golf, field hockey, lacrosse, surfing, Quidditch, contact Live Action Role Play (LARPing), kayak polo, ultimate frisbee, paintball, etc.

Respondents were asked to complete a number of psychometric assessments selected to capture aspects of their thoughts and feelings in general and relative to roller derby: the Big Five Inventory 2 negative emotionality items [BFI 2-Ne; (50)], Multidimensional Scale of Perceived Social Support [MSPSS; (51)], Brief Resilience Scale [BRS; (52)], Sports Mental Toughness Questionnaire [SMTQ; (53)], Injustice Experience Questionnaire [IEQ; (54)], and an adaptation of the Revised Illness Perception Questionnaire [IPQ-R; (55)], referencing concussion. These tools target sources of risk for poor injury recovery. The BFI-2 examines differences in personality and temperament by having respondents rate their level of agreement with 60 statements about themselves (e.g., "I am someone who... worries a lot") on a 5-point scale. T-scores were calculated from the mean rating on the 12 items that make up the Ne subscore (50), with higher values indicating greater negative emotionality. The MSPSS examines the strength of supportive relationships, another potential risk factor for poor response to trauma. Respondents rate their level of agreement with 12 statements on a 7-point scale and a mean score is used for interpretation (1-2.9: low support, 3-5: moderate, 5.1-7: high). The BRS consists of 6 statements respondents rate for agreement on a 5-point scale. Higher mean rating is associated with greater resilience. The SMTQ examines mental toughness in three factors: confidence, constancy, and control using 14 statements, each rated on a $0-3$ scale. Ratings are summed for interpretation (maximum score $=42$ ), with higher toughness associated with higher scores. The IEQ captures how frequently an individual has feelings of severe and irreparable injury-related loss, unfairness, and external attribution of blame, following an injury (54). Respondents rate how frequently they experience 12 thoughts or feelings when thinking about injuries from "Never" 0 to "All the time" 4 (maximum score $=48$ ), with higher scores associated with a more negative outlook. The 35-item IPQ-R captures participants' responses to injury across time, consequences, treatment, and feelings of control through their ratings of agreement with statements about these facets of experience on a 5-point scale, with higher scores associated with poorer adjustment.

Finally, athletes completed an extensive battery of cognitive and linguistic tasks designed to capture areas of weakness following concussion. These data were described elsewhere (56) and raw data are publicly available in the TBIBank (57).

\section{Statistical Analysis}

All statistical analyses were conducted in IBM SPSS Statistics version 28. Reported concussions and orthopedic injuries were counted and calculated out of a standard 1,000 players. Current and past players were examined separately, as it was of interest to consider what conditions were associated with departure from the sport. Demographic variables, participation characteristics, concussion history, and psychometric assessments were examined using t-tests, except in cases where assumptions of normality were violated, in which case corrected calculations are noted. Correlations (Pearson's $r$ ) are reported as they further describe associations among key facets of interest, though these are provided for exploratory purposes only.

In order to examine risk of concussion, we considered three previously-examined salient features of roller derby 


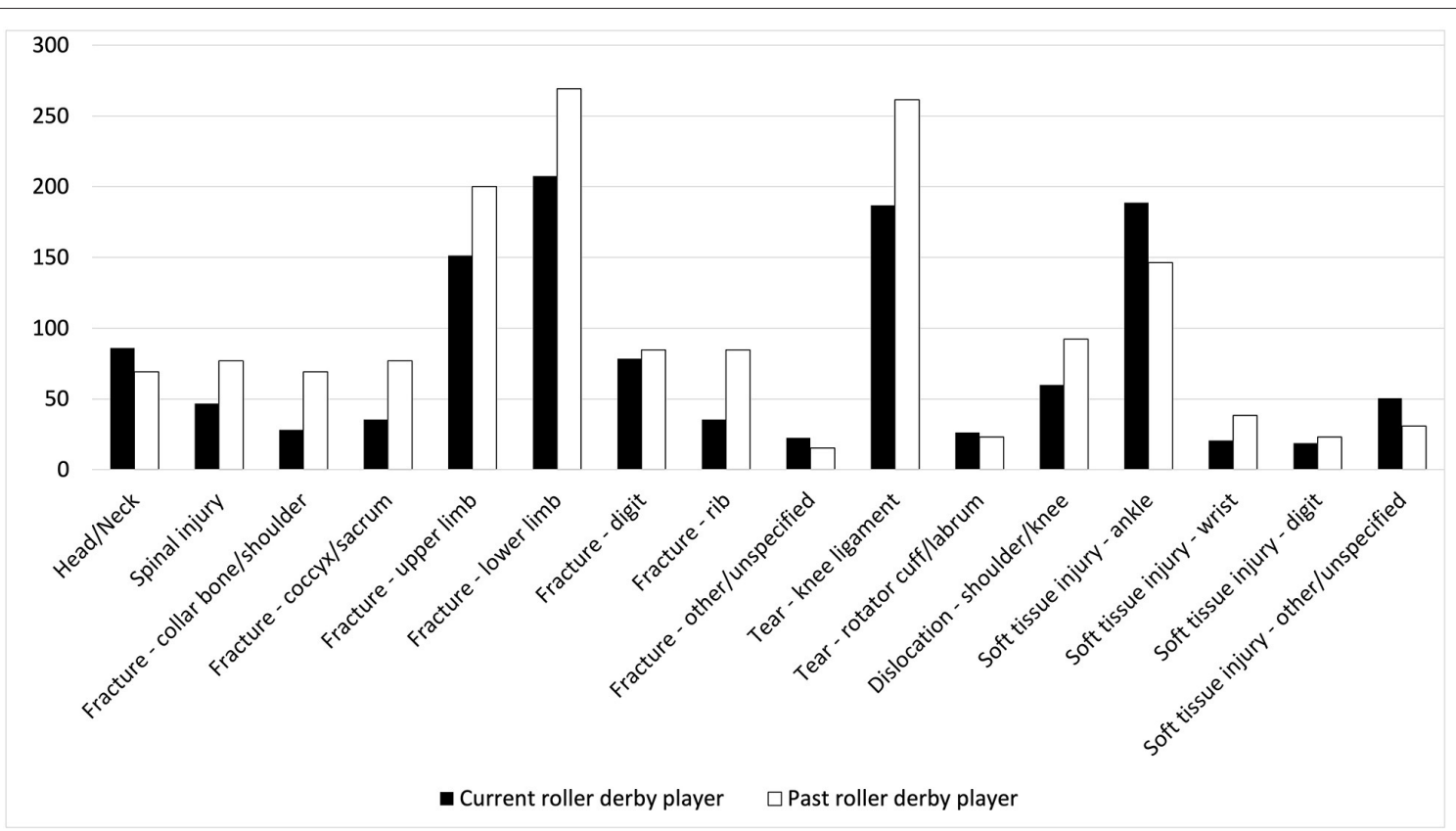

FIGURE 2 | Orthopedic injury history endorsement during roller derby per 1,000 athletes. Head/Neck: whiplash, skull fracture, broken nose; digit: fingers, toes; upper limb: arm, elbow, wrist, hand; lower limb: hip, pelvis, leg, ankle, foot; Soft tissue injury: tears, sprains, dislocation.

participation: the position of the player, frequency of full-contact scrimmages, and kind of flooring played on most frequently (10). While it was initially planned that each of these would be examined using analyses of variance, position was instead analyzed using independent $\mathrm{t}$-tests, as many athletes stated they played multiple positions (thus, the categories were not mutually exclusive). Next, important differences in demographics (age and years of play) as well as self-reported medical history: childhood developmental diagnoses (learning disability, ADD/ADHD, speech-language disorder, fluency disorder, dyslexia), significant neurological history (i.e., reported incidents of neurological conditions more severe than concussion, such as moderateserious TBI and epilepsy), and visual disturbance were entered into regression analyses to arrive at two models.

\section{RESULTS}

\section{Sample Description}

The 665 roller derby players who responded to the survey are described in Table 1. Former roller derby players were older than current players $(\mathrm{t}(663)=3.99, p<0.001)$ and had played significantly longer, by approximately 1 year (Levene's $\mathrm{F}=4.10$, $p=0.04 ; \mathrm{t}(149.11)=2.97, \mathrm{p}=0.004)$. Players engaged in a similar number of practices per month $(\mathrm{t}(660)=0.16, p=0.872)$ and bouts per year (Levene's $\mathrm{F}=10.55=0.001$; $\mathrm{t}$ (133.04) $=$ $1.19, p=0.235$ ). Two-thirds of athletes played a single position, while the remainder switched between roles (block: 69.92\%, jam: $37.41 \%$, pivot: $24.24 \%$ ). Over $80 \%$ of athletes said that all scrimmages were full contact. Many players noted anecdotally that injuries occurred during practice, not bouts. The majority of play was on "sport court," an engineered wood flooring product recommended by the WFTDA, or wood (57.7\%), while just over a third played most frequently on concrete. Derby players frequently also participated in a variety of other sports; 139 current players (26\%) and 43 past players (33\%) played at least one additional sport (Figure 1). Past players reported significantly lower resilience than current players of roller derby (BRS; $\mathrm{t}(455)=3.66, p<0.001)$. They expressed similar levels of dispositional negativity (BFI 2-Ne), social support (MSPSS), mental toughness (SMTQ), perceived injustice following injury (IEQ), and perception of injury (IPQ-R).

Per 1000 athletes, 775.70 concussions were reported among current and 853.85 among former roller derby players (790.98 overall), not accounting for suspected concussions. Only $9 / 34$ recent concussions were that individual's first concussion; the majority had at least one prior concussion. Current and former players described similar numbers of significant hits (Levene's $\mathrm{F}=33.69, p<0.001 ; \mathrm{t}(112.15)=1.51, p=$ $0.13)$. All athletes endorsed substantial orthopedic injury history (Figure 2). Former players reported more concussions than current players (Figure 3 ), by approximately one (Levene's $\mathrm{F}$ $=14.14, p<0.001 ; \mathrm{t}(154.94)=3.72, p<0.001, \mathrm{~d}=0.41)$. However, this was no longer significant when adjusted for the number of years individuals had played (concussions/years; $\mathrm{t}$ $(662)=0.79, p=0.428)$. Higher numbers of concussions were associated significantly with greater mental toughness $(\mathrm{r}=0.15$, $p=0.005)$, and perceptions of injury $(\mathrm{r}=0.17, p=0.002)$ and showed weaker evidence of association with dispositional negativity $(\mathrm{r}=0.12, p=0.03)$ and perceived injustice following injury $(\mathrm{r}=0.28, p=0.04)$. 


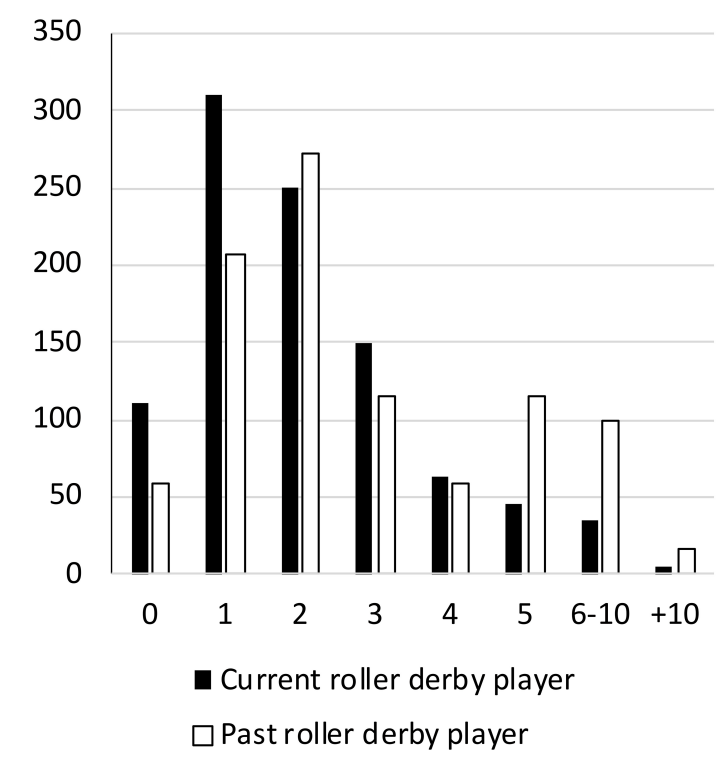

FIGURE 3 | Number of concussions per player during roller derby per 1,000 athletes.

TABLE 2 | Concussions by bout characteristics.

\begin{tabular}{lcc}
\hline & Concussions & \multicolumn{1}{c}{ Test statistic } \\
\hline $\begin{array}{l}\text { Position } \\
\text { Jammer }\end{array}$ & $2.39 \pm 2.31$ & $\mathrm{t}(574)=0.360, p=0.710$ \\
Blocker & $2.4 \pm 2.07$ & $\mathrm{t}(574)=0.929, p=0.353$ \\
Pivot & $2.23 \pm 1.55$ & $\mathrm{t}(421)=0.986, p=0.325$ \\
Court & & $\mathrm{F}(4,569)=1.409, p=0.230$ \\
Wood & $2.52 \pm 2.1$ & \\
Cement & $2.44 \pm 2.34$ & \\
Sport court & $2.15 \pm 1.79$ & \\
Rubber/Plastic & $2.31 \pm 1.90$ & \\
Other/Unknown & $1.69 \pm 1.08$ & \\
Full-Contact scrimmages & & $\mathrm{t}(569)=0.471, p=0.638$ \\
$\quad$ Always & $2.34 \pm 2.10$ & \\
Sometimes & $2.48 \pm 2.09$ & \\
\hline
\end{tabular}

All statistics are two-tailed.

To explore risk of concussion, we considered three salient features of roller derby participation: the position of the player, frequency of full-contact scrimmages, and kind of flooring played on most frequently using $t$-tests and analysis of variance. None of these were statistically associated with greater numbers of concussions among players (Table 2), which hovered consistently around two concussions.

Next, age and years of roller derby participation were entered into a hierarchical multiple regression analysis predicting the total number of concussions among roller derby players (Model 1) as these were demographic features with strong anticipated (and demonstrated) associated with concussions (age; $\mathrm{r}=$ $0.123, p=0.003$; years: $r=0.113, p=0.006$ ), followed by the addition of clinical comorbidities divided into developmental
TABLE 3 | Concussions by demographic and medical history characteristics.

\begin{tabular}{lcc}
\hline & Concussions & Test statistic \\
\hline $\begin{array}{l}\text { Developmental disability } \\
\text { Endorsed }\end{array}$ & $\mathrm{t}(574)=2.20, p=0.03, \mathrm{~d}=0.84$ \\
Not endorsed & $4.07 \pm 2.59$ & \\
Neurological history & $2.33 \pm 2.08$ & \\
Endorsed & $3.70 \pm 2.96$ & \\
Not endorsed & $2.29 \pm 2.03$ & \\
Visual disturbance & & $\mathrm{t}(36)=2.25, p=0.034, \mathrm{~d}=0.68^{\dagger}$ \\
Endorsed & $3.51 \pm 2.97$ & \\
Not endorsed & $2.27 \pm 2.01$ & \\
&
\end{tabular}

All statistics are two-tailed.

${ }^{\dagger}$ Degrees of freedom corrected, as equal variances assumption violated. Data are presented as counts or mean \pm standard deviation.

diagnoses, significant neurological history, and reported uncorrected visual disturbance coded dichotomously (Model 2; Table 3).

The first model was significant in predicting total number of concussions $\left(\mathrm{R}^{2}=0.02, \mathrm{~F}(2,572)=6.52, p=0.002\right)$, with both age $(\mathrm{t}=2.35, p=0.02, \beta=0.03)$ and years of roller derby participation $(\mathrm{t}=2.08, \mathrm{p}=0.04, \beta=0.06)$ contributing significantly to the prediction. The second model also was significant $\left(\mathrm{R}^{2}=0.07, \mathrm{~F}(5,569)=7.85, p<0.001\right)$ and improved on the previous model $(\mathrm{F}(3,569)=8.57, p<0.001)$. Age remained a significant predictor $(t=2.02, p=0.04, \beta=0.02)$, as did years of participation $(\mathrm{t}=1.99, p=0.05, \beta=0.05)$ when using the second model. Among clinical comorbidities, significant neurological history $(\mathrm{t}=3.16,=0.002, \beta=1.38)$ and visual disturbance $(\mathrm{t}=3.58, p<0.001, \beta=1.28)$ were significant, whereas a history of developmental disability was not $(\mathrm{t}=1.89$, $p=0.06, \beta=1.47)$. These factors were associated with greater influence over total number of concussions than years of play or age.

\section{DISCUSSION}

In this study, we investigated the prevalence of concussion and other injuries in roller derby and examined risk factors for injury in the sport. We also explored the affective risk and protective factors of poor injury recovery among players.

Concussions are quite common in roller derby. By our estimation in the present study, most players get one or two concussions during their time in the sport. Only around $10 \%$ of current roller derby players had not had a concussion, and that figure nearly halved by players' departure. These findings answer a slightly different question than the one pursued by Cathorall and Peachey (12), who found that $4 \%$ of players had a concussion in the past year. Our estimate of players who had a concussion in the past 30 days was 6\% (31/535), suggesting that estimates of concussions within the year would be much higher. Our estimates also were higher than those from Hoskins \& Hooker (13). There are a number of potential 
explanations for this discrepancy. Advertisements for this study specifically referenced concussion in roller derby and may have resulted in an over-estimate of concussion in the sport. However, concussion education within the leagues has expanded greatly each year, as have guidelines for sideline concussion monitoring and care. Thus, earlier estimates may have under-appreciated the rate of concussion in the sport. Fractured fingers, toes, upper limb and ankle bones, torn knee ligaments, and ankle sprains and tears were the most commonly reported orthopedic injuries experienced by around a fifth of current players. This is grossly consistent with the findings reported in Pauelsen (10), who found sprains, torn knee ligaments, and concussions as most frequent.

We also explored how dimensions of personality and temperament associated with differences in the patterns of injury among players. While past players were significantly less resilient, they rated themselves like current players in all other examined dimensions - negative emotionality, social support, toughness, perceived injustice, and self-perception with injury. This is concordant with prior work on the culture of roller derby as a sport that tends to attract individuals that are very diverse in certain ways, but tend to share certain values and other traits (24). The number of concussions was associated with greater endorsement of toughness, injury perception, perceived injustice following injury, and a tendency toward negative outlook. More work is needed to understand these relationships, but they may reflect the same underlying effect described by Madrigal et al. (43). The authors found that injured roller derby players, unlike comparable rugby players, were more likely to feel isolated, restless, and cheated after injury and perceived these injuries more negatively in general, as they presented a threat to continued membership in the "sisterhood" that roller derby represents. Injury presented a significant psychosocial and emotional risk to derby players. The authors suggest this may incentivize roller derby players to rejoin play before they are fully healed, thus contributing to more severe, compounded injuries. Policing this behavior in regards to concussion is more complicated than with orthopedic injury, and strategies athletes may use to return to play and rejoin their community despite concussion (i.e., "sandbagging") are well documented among athletes in many other high-pressure sports $(58,59)$.

These data did not support the complex landscape of playrelated risk factors identified in Pauelsen (10). While Pauelsen also used retrospective online survey methodology, minimal information is available to permit critical evaluation of the discrepancies (60). It appears the data were predominantly from European players, which may not have generalized to the predominantly American players in the present sample. While number of years in play was associated with an increased number of concussions, the number of bouts, nature of contact, flooring type, and position all yielded no relationship with concussion prevalence in our sample. Circumstances of play tend to lead to around two concussions on average for current and past players, with significant variability noted (combined $\mathrm{M}=2.35, \mathrm{SD}=2.09$ ). In contrast, the primary risk factor for increased concussion in our data appears to be the player's own prior medical history, particularly a history of significant neurological events (such as a history of seizures or moderate TBI) or uncorrected visual disruption. Unlike facets of gameplay (flooring, contact during scrimmages, number of bouts per year), which are often linked to participation in a certain league or player position, these facets of medical history are individual in nature. Fortunately, these generally are also known to the player, who can enter these into her own calculus about the acceptability of risk associated with participation in this sport.

Our study is limited as the data collection solely relied on remotely filled surveys from the participants. While some studies using survey methods have followed respondents over multiple sessions, this design only involved a single survey. Athletes who responded may have been an atypical or biased sampling of all roller derby players. Since the study predominantly disseminated through social media, social networks active in league- or interest-oriented groups may fail to include the full diversity of players. Respondents also were not subject to independent verification of their medical information or injury narratives (i.e., we had no direct access to medical records). However, this is not so different from how concussions frequently are diagnosed in the clinic, which is often done on the basis of self-report of subjective symptoms, even when the results of commonly used neuroimaging methods are unremarkable and neurological examination is normal (61). Online data collection is becoming an increasingly common and validated source of health and behavioral data $(44,46,47)$. The design of this study did not permit us to determine if respondents had high intrarater consistency. A weakness of this approach is that there was no practical means of following up with respondents to better understand ambiguous responses, incomplete information, or to ask follow-up questions that arose during analysis (common activities when patients are seen in person). One question that we failed to ask was whether athletes who reported a recent concussion had returned to play or were otherwise considered recovered at the time of the survey. While this has more influence on the cognitive and linguistic dimensions of the survey, it could potentially have impacted the quality of all responses by individuals with recent injuries, as using screens during this time is often challenging and may lead to an increase in symptoms, such as headache and light-sensitivity $(62,63)$.

Roller derby is an exciting sport that holds a position of great importance among many players, well beyond the end of a bout. Discourse samples describing the sport frequently mentioned community, empowerment, and support, echoing the extensive literature on the sport in gender studies and ethnography. We were unable to examine gender diversity and its impact on the sport of roller derby given the small number of participants but appreciate that this is an important direction for future work. Increased attention in recent years has facilitated a richer foundation for players to make informed decisions about participation; however, these findings raise as many questions as are answered, particularly regarding the impact of gender on cognitive sequelae of concussion, departure from the sport, and long-term consequences of injury. It is our hope that medical research will continue to examine this unique sport. 


\section{DATA AVAILABILITY STATEMENT}

The original contributions presented in the study are publicly available. This data can be found here: Stockbridge M. English Stockbridge Narrative \& Roller-Derby elicited written corpora. TBIBank2021. doi: 10.21415/D70T-C409.

\section{ETHICS STATEMENT}

The studies involving human participants were reviewed and approved by University of Maryland Institutional Review Board. The patients/participants provided their written informed consent to participate in this study.

\section{REFERENCES}

1. Giza CC, Kutcher JS, Ashwal S, Barth J, Getchius TS, Gioia GA, et al. Summary of evidence-based guideline update: evaluation and management of concussion in sports: report of the Guideline Development Subcommittee of the American Academy of Neurology. Neurology. (2013) 80:22507. doi: 10.1212/WNL.0b013e31828d57dd

2. Losoi H, Silverberg ND, Wäljas M, Turunen S, Rosti-Otajärvi E, Helminen M, et al. Recovery from mild traumatic brain injury in previously healthy adults. J Neurotrauma. (2016) 33:766-76. doi: 10.1089/neu.2015.4070

3. Pellman EJ, Lovell MR, Viano DC, Casson IR. Concussion in professional football: recovery of NFL and high school athletes assessed by computerized neuropsychological testing-part 12. Neurosurgery. (2006) 58:263-74. doi: 10.1227/01.NEU.0000200272.56192.62

4. Stone S, Lee B, Garrison JC, Blueitt D, Creed K. Sex differences in time to return-to-play progression after sport-related concussion. Sports Health. (2017) 9:41-4. doi: 10.1177/1941738116672184

5. Baker JG, Leddy JJ, Darling SR, Shucard J, Makdissi M, Willer BS. Gender differences in recovery from sports-related concussion in adolescents. Clin Pediatr (Phila). (2016) 55:771-5. doi: 10.1177/0009922815606417

6. Desai N, Wiebe DJ, Corwin DJ, Lockyer JE, Grady MF, Master CL. Factors affecting recovery trajectories in pediatric female concussion. Clin J Sport Med. (2019) 29:361-7. doi: 10.1097/JSM.0000000000000646

7. Master CL, Katz BP, Arbogast KB, McCrea MA, McAllister TW, Pasquina PF, et al. Differences in sport-related concussion for female and male athletes in comparable collegiate sports: a study from the NCAA-DoD Concussion Assessment, Research and Education (CARE) Consortium. Br J Sports Med. (2021) 55:1387-94. doi: 10.1136/bjsports-2020-103316

8. Kearney MC. Tough girls in a rough game: Televising the unruly female athletes of contemporary roller derby. Feminist Media Studies. (2011) 11:283301. doi: 10.1080/14680777.2010.535309

9. Paul J. Sport and bodily empowerment: Female athletes' experiences with roller derby, mixed martial arts, and rugby. Journal of Alternative Perspectives in the Social Sciences. (2015) 6:402-38.

10. Pauelsen M. Roller Derby: A Descriptive Study of the Athletes, Sustained Injuries and Risk Factors. (Bachelor's thesis). Luleå, Sweden: Luleå University of Technology (2013).

11. Lane AD, Berkman MR, Verbunker D, Shekell T, Bouska M, Barnett L, et al. Retrospective chart analysis of concussion discharge instructions in the emergency department. J Emerg Med. (2017) 52:690-8. doi: 10.1016/j.jemermed.2016.12.017

12. Cathorall ML, Peachey AA. Incidence and predictors of roller derby injuries among female roller derby athletes. Int J Injury Control Safe Promot. (2018) 25:387-92. doi: 10.1080/17457300.2018.1431946

13. Hoskins AL, Hooker RS. Sudden impact: concussion in female roller derby athletes. Ann Sports Med Res. (2015) 2:1041. doi: 10.1097/01.JAA.0000471292.02985.6a

14. Noble JM, Hesdorffer DC. Sport-related concussions: a review of epidemiology, challenges in diagnosis, and potential risk factors. Neuropsychol Rev. (2013) 23:273-84. doi: 10.1007/s11065-013-9239-0

\section{AUTHOR CONTRIBUTIONS}

MS and RN conceived of the study and design. MS acquired the data, organized the data for analysis, and wrote the first draft of the manuscript. ZK and MS arrived at a statistical plan and performed the statistical analysis. All authors contributed to the manuscript revision and read and approved the submitted version.

\section{FUNDING}

MS was supported by NIDCD P50 DC014664.

15. Lynall RC, Mihalik JP, Pierpoint LA, Currie DW, Knowles SB, Wasserman EB, et al. The first decade of web-based sports injury surveillance: descriptive epidemiology of injuries in US high school boys' ice hockey (2008-2009 Through 2013-2014) and National Collegiate Athletic Association Men's and Women's Ice Hockey (2004-2005 Through 2013-2014). J Athl Train. (2018) 53:1129-42. doi: 10.4085/1062-6050-176-17

16. Casson IR, Viano DC, Powell JW, Pellman EJ. Twelve years of National Football League concussion data. Sports Health. (2010) 2:471-83. doi: 10.1177/1941738110383963

17. Guskiewicz KM, Bruce SL, Cantu RC, Ferrara MS, Kelly JP, McCrea M, et al. National Athletic Trainers' Association position statement: management of sport-related concussion. J Athl Train. (2004) 39:280.

18. Crisco JJ, Greenwald RM. Let's get the head further out of the game: a proposal for reducing brain injuries in helmeted contact sports. Curr Sports Med Rep. (2011) 10:7. doi: 10.1249/JSR.0b013e318205e063

19. Marar M, McIlvain NM, Fields SK, Comstock RD. Epidemiology of concussions among United States high school athletes in 20 sports. Am J Sports Med. (2012) 40:747-55. doi: 10.1177/0363546511435626

20. Graham R, Rivara FP, Ford MA, Spicer CM, Youth CoS-RCi, Council NR. Sports-related concussions in youth: improving the science, changing the culture. Washington, DC: National Academies Press (US) (2014).

21. Women's Flat Track Derby Association. WFTDA Risk Management Guidelines. Available online at: https://staticwftdacom/insurance/wftda-riskmanagement-guidelinespdf2020 (accessed January 1, 2022).

22. Mrazik M, Naidu D, Lebrun C, Game A, Matthews-White J. Does an individual's fitness level affect baseline concussion symptoms? J Athl Train. (2013) 48:654-8. doi: 10.4085/1062-6050-48.3.19

23. Storms CE. There's no sorry in roller derby": a feminist examination of identity of women in the full contact sport of roller derby'. N Y Sociol. (2008) 3:68-87.

24. Beaver TD. "By the skaters, for the skaters" The DIY ethos of the roller derby revival. J Sport Soc Iss. (2012) 36:25-49. doi: 10.1177/01937235114 33862

25. Carlson J. The female signifiant in all-women's amateur roller derby. Sociol Sport J. (2010) 27:428-40. doi: 10.1123/ssj.27.4.428

26. Eklund A, Masberg BA. Participation in roller derby, the influence on body image. Clothing Textiles Res J. (2014) 32:49-64. doi: 10.1177/0887302X13511295

27. Pavlidis A, Fullagar S. Becoming roller derby grrrls: Exploring the gendered play of affect in mediated sport cultures. Int Rev Sociol Sport. (2013) 48:67388. doi: 10.1177/1012690212446451

28. Pavlidis A, Fullagar S. Sport, Gender and Power: The Rise of Roller Derby. London: Routledge (2016). doi: 10.4324/9781315610429

29. Parry DC. "Skankalicious": Erotic capital in women's flat track roller derby. Leisure Sci. (2016) 38:295-314. doi: 10.1080/01490400.2015.1113149

30. Liu HL, Bradley MJ, Burk B. I am roller derby: the serious leisure and leisure identity of roller derby participants. World Leis J. (2016) 58:2843. doi: 10.1080/16078055.2015.1083466

31. Klein U. Beyond the fishnets: female empowerment through roller derby. IJFAB. (2016) 9:198-207. doi: 10.3138/ijfab.9.2.198 
32. Watson D, Clark LA. Negative affectivity: the disposition to experience aversive emotional states. Psychol Bull. (1984) 96:465-90. doi: 10.1037/0033-2909.96.3.465

33. Watson D, Clark LA, McIntyre CW, Hamaker S. Affect, personality, and social activity. J Pers Soc Psychol. (1992) 63:1011-25. doi: 10.1037/0022-3514.63.6.1011

34. Caspi A, Roberts BW, Shiner RL. Personality development: Stability and change. Annu Rev Psychol. (2005) 56:45384. doi: 10.1146/annurev.psych.55.090902.141913

35. Barlow DH, Sauer-Zavala S, Carl JR, Bullis JR, Ellard KK. The nature, diagnosis, and treatment of neuroticism: Back to the future. Clin Psychol Sci. (2013) 2:344-65. doi: 10.1177/2167702613505532

36. Ponsford J, Cameron P, Fitzgerald M, Grant M, Mikocka-Walus A, Schönberger M. Predictors of postconcussive symptoms 3 months after mild traumatic brain injury. Neuropsychology. (2012) 26:304. doi: 10.1037/a0027888

37. Vargas G, Rabinowitz A, Meyer J, Arnett PA. Predictors and prevalence of postconcussion depression symptoms in collegiate athletes. J Athl Train. (2015) 50:250-5. doi: 10.4085/1062-6050-50.3.02

38. Silverberg ND, Gardner AJ, Brubacher JR, Panenka WJ, Li JJ, Iverson GL. Systematic review of multivariable prognostic models for mild traumatic brain injury. J Neurotrauma. (2015) 32:517-26. doi: 10.1089/neu.2014. 3600

39. Shackman AJ, Tromp DP, Stockbridge MD, Kaplan CM, Tillman RM, Fox AS. Dispositional negativity: an integrative psychological and neurobiological perspective. Psychol Bull. (2016) 142:1275. doi: 10.1037/bul000 0073

40. Zimet GD, Powell SS, Farley GK, Werkman S, Berkoff KA. Psychometric characteristics of the multidimensional scale of perceived social support. J Pers Assess. (1990) 55:610-7. doi: 10.1080/00223891.1990.9674095

41. Bruwer B, Emsley R, Kidd M, Lochner C, Seedat S. Psychometric properties of the multidimensional scale of perceived social support in youth. Compr Psychiatry. (2008) 49:195-201. doi: 10.1016/j.comppsych.2007.09.002

42. Petrie TA, Deiters J, Harmison RJ. Mental toughness, social support, and athletic identity: Moderators of the life stress-injury relationship in collegiate football players. Sport, Exerc Perform Psychol. (2014) 3:13. doi: $10.1037 / \mathrm{a} 0032698$

43. Madrigal L, Wurst K, Gill DL. The role of mental toughness in coping and injury response in female roller derby and rugby athletes. J Clin Sport Psychol. (2016) 10:137-54. doi: 10.1123/JCSP.2015-0021

44. Barak A, English N. Prospects and limitations of psychological testing on the Internet. J Technol Hum Serv. (2002) 19:65-89. doi: 10.1300/J017v19n02_06

45. Chuah SC, Drasgow F, Roberts BW. Personality assessment: does the medium matter? No J Res Pers. (2006) 40:359-76. doi: 10.1016/j.jrp.2005.01.006

46. Templer KJ, Lange SR. Internet testing: equivalence between proctored lab and unproctored field conditions. Comput Human Behav. (2008) 24:121628. doi: 10.1016/j.chb.2007.04.006

47. Illingworth AJ, Morelli NA, Scott JC, Boyd SL. Internet-based, unproctored assessments on mobile and non-mobile devices: Usage, measurement equivalence, and outcomes. J Bus Psychol. (2015) 30:325-43. doi: 10.1007/s10869-014-9363-8

48. Lewandowski L, Rieger B, Smyth J, Perry L, Gathje R. Measuring post-concussion symptoms in adolescents: Feasibility of ecological momentary assessment. Arch Clin Neuropsychol. (2009) 24:7916. doi: 10.1093/arclin/acp087

49. King N, Crawford S, Wenden F, Moss N, Wade D. The rivermead post concussion symptoms questionnaire: a measure of symptoms commonly experienced after head injury and its reliability. J Neurol. (1995) 242:58792. doi: 10.1007/BF00868811

50. Soto CJ, John OP. The next Big Five Inventory (BFI-2): Developing and assessing a hierarchical model with 15 facets to enhance bandwidth, fidelity, and predictive power. J Pers Soc Psychol. (2017) 113:11743. doi: $10.1037 /$ pspp0000096

51. Zimet GD, Dahlem NW, Zimet SG, Farley GK. The multidimensional scale of perceived social support. J Pers Assess. (1988) 52:3041. doi: 10.1207/s15327752jpa5201_2

52. Smith BW, Dalen J, Wiggins K, Tooley E, Christopher P, Bernard J. The brief resilience scale: assessing the ability to bounce back. Int J Behav Med. (2008) 15:194-200. doi: 10.1080/10705500802222972

53. Sheard M, Golby J, Van Wersch A. Progress toward construct validation of the Sports Mental Toughness Questionnaire (SMTQ). Eur J Psychol Assess. (2009) 25:186-93. doi: 10.1027/1015-5759.25.3.186

54. Sullivan MJ, Adams H, Horan S, Maher D, Boland D, Gross R. The role of perceived injustice in the experience of chronic pain and disability: scale development and validation. J Occup Rehabil. (2008) 18:24961. doi: 10.1007/s10926-008-9140-5

55. Moss-Morris R, Weinman J, Petrie K, Horne R, Cameron L, Buick D. The revised illness perception questionnaire (IPQ-R). Psychol Health. (2002) 17:116. doi: 10.1080/08870440290001494

56. Stockbridge MD, Keser Z, Newman R. Concussion in women's flat-track roller derby. In: Top Scoring Oral presentation at the 14th World Congress on Brain Injury, International Brain Injury Association, (2021).

57. Stockbridge M. English Stockbridge Narrative \& Roller-Derby Elicited Written Corpora. TBIBank (2021). doi: 10.21415/D70T-C409

58. Erdal K. Neuropsychological testing for sports-related concussion: how athletes can sandbag their baseline testing without detection. Arch Clin Neuropsychol. (2012) 27:473-9. doi: 10.1093/arclin/acs050

59. Rizzo J-R, Hudson TE, Martone J, Dai W, Ihionu O, Chaudhry Y, et al. How sandbag-able are concussion sideline assessments? A close look at eye movements to uncover strategies. Brain Inj. (2021) 35:42635. doi: 10.1080/02699052.2021.1878554

60. Pauelsen AM, Jacobson I, Larsson A, Tegner Y. Skadepanorama och riskfaktorer inom Flat Track Roller Derby. Svensk Idrottsmedicin. (2014) 2014:22-5.

61. Mannix R, Meehan III WP, Pascual-Leone A. Sports-related concussions-media, science and policy. Nat Rev Neurol. (2016) 12:486-90. doi: 10.1038/nrneurol.2016.99

62. Macnow T, Curran T, Tolliday C, Martin K, McCarthy M, Ayturk D, et al. Effect of screen time on recovery from concussion: a randomized clinical trial. JAMA Pediatr. (2021) 175:1124-31. doi: 10.1001/jamapediatrics.2021.2782

63. Leitch H, Ayers E, Andrews A. A review of concussion management in the young adult; the importance of baseline testing and caregiver education. J Natl Med Assoc. (2015) 107:60-5. doi: 10.1016/S0027-9684(15)30053-5

Conflict of Interest: The authors declare that the research was conducted in the absence of any commercial or financial relationships that could be construed as a potential conflict of interest.

Publisher's Note: All claims expressed in this article are solely those of the authors and do not necessarily represent those of their affiliated organizations, or those of the publisher, the editors and the reviewers. Any product that may be evaluated in this article, or claim that may be made by its manufacturer, is not guaranteed or endorsed by the publisher.

Copyright (C) 2022 Stockbridge, Keser and Newman. This is an open-access article distributed under the terms of the Creative Commons Attribution License (CC BY). The use, distribution or reproduction in other forums is permitted, provided the original author(s) and the copyright owner(s) are credited and that the original publication in this journal is cited, in accordance with accepted academic practice. No use, distribution or reproduction is permitted which does not comply with these terms. 